\title{
An integrable family of Monge-Ampère equations and their multi-Hamiltonian structure $2 k$
}

\author{
Y. Nutku and Ö. Sanoğlu \\ Department of Mathematics, Bilkent University, 06533 Ankara, Turkey
}

\begin{abstract}
Received 14 September 1992; revised manuscript received 2 November 1992; accepted for publication 16 December 1992
\end{abstract} Communicated by A.P. Fordy

\begin{abstract}
We have identified a completely integrable family of Monge-Ampère equations through an examination of their Hamiltonian structure. Starting with a variational formulation of the Monge-Ampère equations we have constructed the first Hamiltonian operator through an application of Dirac's theory of constraints. The completely integrable class of Monge-Ampère equations are then obtained by solving the Jacobi identities for a sufficiently general form of the second Hamiltonian operator that is compatible with the first.
\end{abstract}

Completely integrable nonlinear evolution equations are "universal" and "widely applicable" [1]. It is not surprising, therefore, that there are also very few of them! Still the question remains as to whether or not we are missing any important integrable equations.

One of the major families of nonlinear partial differential equations in $1+1$ dimensions that has not been sufficiently investigated for its possible complete integrability is the Monge-Ampère family

$u_{t i} u_{x x}-u_{t x}^{2}=F$

where $F$ is assumed to be analytic in its arguments which consist of $u$ and its first derivatives. The nonexistence of a linear dispersive part to eqs. (1) is responsible for their exclusion as a candidate from various extensive searches for completely integrable equations [2]. On the other hand the origins of the Monge-Ampère equation in differential geometry suggest that for some particular choices of $F$, eq. (1) may indeed turn out to be completely integrable. In this context we recall that

* In memory of a young mathematician: Koray Karahan, 20 March 1970 - 10 December 1991.

$$
\begin{aligned}
F & =K\left(1+u_{x}^{2} \pm u_{t}^{2}\right)^{2}, \\
& =K, \\
& =\mathrm{e}^{u}, \\
& =u^{-4}, \\
& =f_{1} u_{t t}+f_{2} u_{t x}+f_{3} u_{x x}+f_{4}
\end{aligned}
$$

are familiar examples of Monge-Ampère equations that arise in well-known problems of geometry. Equation (1b) is the original Monge-Ampère equation that describes surfaces of constant curvature $K= \pm 1,0$ which could therefore be gauge-equivalent [3] to either the sine-Gordon or the Liouville equations. The deceptively simpler looking case of eq. (1c) has been studied [4] in connection with heavenly metrics in complex general relativity. Similarly eq. ( $1 \mathrm{~d}$ ) is a $1+1$ version of the equation governing the Kähler potential for the K3 surface of Kummer [5] for which the metric has remained elusive for over a century in spite of its importance as an instanton in quantum gravity [6]. Finally Rogers [7] has pointed out that eq. (1e) is related to the DoddBullough equation through an integrable choice of the equation of state for Eulerian gas dynamics. Martin [8] has given examples of Monge-Ampère systems as reductions of the equations of gas dynamics. Some of these are integrable. Martin's systems 
are of the type of eqs. (1f) where the right hand side is linear in the second derivatives and $f_{i}$ depend on $x, t$ as well as $u$ and its first derivatives.

One of the most direct ways of identifying a completely integrable non-linear partial differential equation consists of an examination of its Hamiltonian structure. In particular, if we can find two independent but compatible non-degenerate Hamiltonian operators for a given equation, then by Magri's theorem [9] it follows that there exists an infinite family of conserved Hamiltonians which are in involution with respect to the generalized Poisson brackets defined in terms of both of these Hamiltonian operators. In general this is a formidable task, but there is an algorithm [10] for constructing the first Hamiltonian operator: The first generalized Poisson bracket is the Dirac bracket [11].

The first step towards a Hamiltonian formulation of any system requires the existence of a Lagrangian. The Monge-Ampère family of equations (1a) are the Euler-Lagrange equations for the variational principle

$\delta I=0, \quad I=\int \mathscr{L} \mathrm{d} t \mathrm{~d} x$,

where the Lagrangian is given by

$\mathscr{L}=\Omega \mathscr{L}_{\mathrm{B}}+\Phi$,

$\mathscr{L}_{\mathrm{B}}=\frac{1}{2}\left[\left(1+u_{x}^{2}\right) u_{t t}-2 u_{t} u_{x} u_{x t}-\left(1-u_{t}^{2}\right) u_{x x}\right]$

and $\Omega, \Phi$ are in general functions of $u$ and its first derivatives which must be specialized according to the desired choice of $F$ on the right hand side of eq. (1a). The crucial part of the Lagrangian that yields a Monge-Ampère operator on the left hand side of eq. (1a) is $\mathscr{L}_{\mathrm{B}}$. This is a second order degenerate Lagrangian and the passage to the Hamiltonian formulation of such degenerate systems requires the use of Dirac's theory of constraints [11]. We note that $\mathscr{L}_{\mathrm{B}}$ happens to be precisely of the form of the BornInfeld equation, i.e. the hyperbolic version of the equation governing minimal surfaces. It is interesting to recall that the Born-Infeld equation itself admits the richest Hamiltonian structure [12] among all the two-component equations of hydrodynamic type [13]. Finally, it can be readily verified that the particular choices

$$
\text { (I) } \begin{array}{ll}
\Omega=\left(1 \pm u_{t}^{2}+u_{x}^{2}\right)^{-1}, & \Phi=K u, \\
\text { (II) } \Omega=1, & \Phi=3 K u, \\
& =3 \mathrm{e}^{u}, \\
& =-u^{-3},
\end{array}
$$

yield eqs. (1b)-(1e) respectively. For $\Omega=1$, eq. (2c) reduces to

$\mathscr{L}_{\mathrm{B}}=\frac{1}{2} u_{x}^{2} u_{t t}-u_{t} u_{x} u_{x t}+\frac{1}{2} u_{t}^{2} u_{x x}$,

which is familiar from surface theory [14]. The symmetries of eqs. (2c) and (2d) are well-known and they can be used to construct Noether currents for the Monge-Ampère equations provided that the choice of $\Omega, \Phi$ is compatible with these symmetries.

In order to pass to a Hamiltonian formulation of the various Lagrangian systems in eqs. ( $2 a$ ) we need to start with an equivalent first order form which depends only on the velocities. Thus we rewrite the Monge-Ampère equations in the form

$u_{t}=q$,

$q_{t}=\frac{1}{u_{x x}}\left(q_{x}^{2}+F\right)$,

appropriate to a pair of evolution equations. We shall henceforth use $u^{i}$ with $i=1,2$ ranging over the variables $u, q$ respectively. For $\Omega=1$ the Lagrangian for the first order form of the Monge-Ampère equations is given by

$\mathscr{L}=\frac{1}{2} u_{x}^{2} q_{t}-u_{x} q_{x} u_{t}+\frac{1}{2} q^{2} u_{x x}+\Phi$,

where $F$ is a gradient with potential $\Phi$. This is manifestly a degenerate Lagrangian as its Hessian vanishes identically. Thus we need to apply Dirac's theory of constraints in order to cast it into canonical form. The details of this process will be discussed elsewhere [15]. Here we shall only record the result that for $\Omega=1$ Dirac's theory yields

$H_{1}=\frac{1}{2} q^{2} u_{x x}+\Phi$

as the Hamiltonian function and the Dirac bracket gives rise to the Hamiltonian operator

$J_{0}=\left(\begin{array}{cc}0 & 1 / u_{x x} \\ -1 / u_{x x} & \left(q_{x} / u_{x x}^{2}\right) \mathrm{D}+\mathrm{D}\left(q_{x} / u_{x x}^{2}\right)\end{array}\right)$,

with $D \equiv \partial / \partial x$. It can be verified that $J_{0}$ satisfies the 
Jacobi identities and the equations of motion (4) are cast into canonical form $u_{t}^{i}=J_{0}^{i k} \delta H_{1} / \delta u^{k}$. Furthermore $J_{0}$ is a non-degenerate Hamiltonian operator with the inverse

$K_{0}=\left(\begin{array}{cc}q_{x} \mathrm{D}+\mathrm{D} q_{x} & -u_{x x} \\ u_{x x} & 0\end{array}\right)$

so that an alternative statement of Hamiltonian structure [16] is provided by the symplectic twoform $\omega=\int \mathrm{d} u^{i} K_{i j} \mathrm{~d} u^{j} \mathrm{~d} x$ given by

$\omega_{0}=\int\left(q_{x} \mathrm{~d} u \wedge \mathrm{d} u_{x}-u_{x x} \mathrm{~d} u \wedge \mathrm{d} q\right) \mathrm{d} x$,

which can be readily verified to be a closed two-form.

The Hamiltonian operator (6), or its symplectic counterpart (7), is applicable to a wide variety of Monge-Ampère equations but it is not by any means sufficient to show the integrability of any one of them. For this purpose we need to find at least a bi-Hamiltonian structure. That is, a second Hamiltonian operator $J_{1}$ such that the Lenard-Magri recursion relation

$u_{t_{n+1}}^{i}=J_{0}^{i k} \frac{\delta}{\delta u^{k}} H_{n+1}=J_{1}^{i k} \frac{\delta}{\delta u^{k}} H_{n}, \quad n=0,1,2, \ldots$,

is satisfied. The clue to the possible existence of $J_{1}$ and the infinitely many conserved Hamiltonians $H_{n}$ comes from the observation that for all equations of the type of eq. (1a)

$H_{0}=-\frac{1}{2} u_{x}^{2} q_{x}$

is conserved. This can be inferred from the flux of $H_{1}$ in eq. (5) and the symmetry between $x, t$ in eqs. (1a). The knowledge of $H_{0}$ provides crucial information on the structure of $J_{1}$ by enabling us to concentrate on the $n=0$ case of eqs. (8). Then the Jacobi identities determine which particular equation among eqs. (1a) will admit a bi-Hamiltonian structure.

In order to simplify the discussion of the second Hamiltonian operator we shall first restrict our attention to

$u_{t t} u_{x x}-u_{t x}^{2}=0$,

which will be referred to as the "homogeneous" Monge-Ampère equation. In this case it can be readily verified that
$J_{1}=\left(\begin{array}{cc}0 & q / u_{x} u_{x x} \\ -q / u_{x} u_{x x} & \left(q q_{x} / u_{x} u_{x x}^{2}\right) \mathrm{D}+\mathrm{D}\left(q q_{x} / u_{x} u_{x x}^{2}\right)\end{array}\right)$

is the second Hamiltonian operator. It satisfies the Jacobi identities and eq. (10) can be expressed as a Hamiltonian system in two different ways through eqs. (8). Furthermore $J_{0}, J_{1}$ are compatible Hamiltonian operators since $J_{0}+\mu J_{1}$ with $\mu$ an arbitrary constant also satisfies the Jacobi identities. Then, by Magri's theorem, we have infinitely many Hamiltonians,

$H_{0}=\frac{1}{2} u_{x}^{2} q_{x}$,

$H_{1}=q q_{x} u_{x}$,

$H_{2}=\frac{1}{2} q^{2} q_{x} \ln u_{x}$,

$H_{3}=\frac{1}{3 u_{x}} q^{3} q_{x}, \quad \ldots$,

which are in involution with respect to Poisson brackets defined by both $J_{0}$ and $J_{1}$. Equations ( 9 ) suggest that for an arbitrary differentiable function $f$, the quantity

$H=q^{2} q_{x} f\left(q / u_{x}\right)$

is conserved by virtue of the homogeneous MongeAmpère equation and this is readily verified.

The symplectic two-form that corresponds to the second Hamiltonian operator for the homogeneous Monge-Ampère equation is obtained by inverting $J_{1}$ which yields

$K_{1}=\left(\begin{array}{cc}\left(u_{x} q_{x} / q\right) \mathrm{D}+\mathrm{D}\left(u_{x} q_{x} / q\right) & -u_{x} u_{x x} / q \\ u_{x} u_{x x} / q & 0\end{array}\right)$

and we have the closed two-form

$\omega_{1}=\int\left(\frac{u_{x} q_{x}}{q} \mathrm{~d} u \wedge \mathrm{d} u_{x}-\frac{u_{x} u_{x x}}{q} \mathrm{~d} u \wedge \mathrm{d} q\right) \mathrm{d} x$.

Thus in eqs. ( $7 \mathrm{~b})$ and $(12 \mathrm{~b})$ we have a symplectic pair. The existence of the inverses of the Hamiltonian operators (6) and (11) is sufficient to establish their non-degeneracy so that the conditions of Magri's theorem are fulfilled. Since we have a nondegenerate pair of compatible Hamiltonian operators for the homogeneous Monge-Ampère equation 
we can assert its complete integrability [17].

Knowledge of the Hamiltonian and symplectic operators for the homogeneous Monge-Ampere equation (10) enables us to obtain the recursion operator [18] through $\mathscr{R}=J_{1} J_{0}^{-1}$. We find that it is a local operator given by

$\mathscr{R}=\left(\begin{array}{cc}q / u_{x} & 0 \\ q_{x}^{2} / u_{x} u_{x x}-q q_{x} / u_{x}^{2} & q / u_{x}\end{array}\right)$

and satisfies

$\mathscr{R}_{t}=[\mathscr{A}, \mathscr{R}]$,

where

$\mathscr{A}=\left(\begin{array}{cc}0 & 1 \\ -\left(q_{x}^{2} / u_{x x}^{2}\right) \mathrm{D}^{2} & 2\left(q_{x} / u_{x x}\right) \mathrm{D}\end{array}\right)$.

All the higher flows obtained by an application of the recursion operator to the Monge-Ampère flow (4) yield the homogeneous Monge-Ampère equation (10) itself up to a weight factor which is $u_{x}^{n}$ for the $t_{n+1}$ flow.

We have shown that eq. (10) admits a bi-Hamiltonian structure. Guided by this example we can find a class of Monge-Ampère equations which admits a second Hamiltonian operator compatible with the first. Such a Hamiltonian operator will be given in terms of two functions $L, M$ entering into the ansatz

$J=\left(\begin{array}{cc}0 & L \\ -L & M \mathrm{D}+\mathrm{D} M\end{array}\right)$

and the Jacobi identities enable us to determine the explicit dependence of $L, M$ on $u_{x x}, q_{x}$. The result

$L=\frac{1}{a u_{x x}+b}$,

$M=\frac{a q_{x}+c}{\left(a u_{x x}+b\right)^{2}}$

contains three functions $a, b, c$ of three variables, namely $u$ and its first derivatives $u_{x}, q$, which apart from the single requirement

$a_{u} u_{x}=b_{u x}+c_{q}$

can be chosen arbitrarily. The appearance of these arbitrary functions in the Hamiltonian operator (14) provides us with many opportunities for constructing Monge-Ampère equations with multi-Hamilto- nian structure. For example, it can be verified that the special case

$a=\left(1+\mu q / u_{x}\right)^{-1}, \quad b=c=0$

yields our earlier results for the bi-Hamiltonian structure of the homogeneous Monge-Ampere equation (10).

We must emphasize that the ansatz in eq. (14a) does not yield the most general form of the second Hamiltonian operator compatible with the first. Equations (14) describe a manageable but rather restricted class of Hamiltonian operators appropriate to a family of integrable Monge-Ampère equations. With the important exception of the Martin systems in eqs. (1f), most of the interesting equations we have discussed in eqs. (1a) are not in this class and further work is required in order to draw conclusions about their integrability. Perhaps one of the simplest equations in the integrable category we have obtained above is given by

$u_{t t} u_{x x}-u_{t x}^{2}=u_{x}^{-1}\left[u_{t}^{2} h\left(u, u_{x}\right)\right]_{x}$,

where $h$ is an arbitrary function of its arguments. Evidently the family of integrable Monge-Ampère equations is much larger than its formidable looking nonlinearity would suggest.

This work was in part supported by the scientific research council of Turkey, TÜBİTAK, under TBAG/ÇG-1.

\section{References}

[1] F. Calogero, in: What is integrability?, ed. V.E. Zakharov (Springer, Berlin, 1991).

[2] H. Flaschka; A.C. Newell and M. Tabor; and A.V. Mikhailov, A.B. Shabat and V.V. Sokolov, in: What is integrability?, ed. V.E. Zakharov (Springer, Berlin, 1991).

[3] L.D. Fadeev and L.A. Takhtajan, Hamiltonian methods in the theory of solitons (Springer, Berlin, 1988).

[4] C.P. Boyer and J.F. Plebanski, J. Math. Phys. 18 (1977) 1022.

[5] S.T. Yau, Proc. Natl. Acad. Sci. USA 74 (1977) 1798; Commun. Pure Appl. Math. 31 (1978) 339.

[6] D.N. Page, Phys. Lett. B 80 (1978) 55.

[7] C. Rogers, private communication.

[8] M.H. Martin, Can. J. Math. 5 (1953) 37; Q. Appl. Math. 8 (1951) 137. 
[9] F. Magri, J. Math. Phys. 19 (1978) 1156;

F. Magri, C. Morosi and O. Ragnisco, Commun. Math. Phys. 99 (1985) 115;

P.J. Olver, Graduate texts in mathematics, Vol. 107. Applications of Lie Groups to differential equations (Springer, Berlin, 1986).

[10] Y. Nutku, J. Math. Phys. 25 (1984) 2007;

M.J. Bergvelt and E.A. DeKerf, Lett. Math. Phys. 10 (1985) 13 ;

F. Lund, Physica D 18 (1986) 420.

[11] P.A.M. Dirac, Belfer Graduate School of Science monographs series 2. Lectures on quantum mechanics (New York, 1964);
A. Hanson, T. Regge and C. Teitelboim, Acad. Naz. Lincei (Rome, 1976).

[12] M. Ark, F. Neyzi, Y. Nutku, P.J. Olver and J. Verosky, J. Math. Phys. 30 (1988) 1338.

[13] B.A. Dubrovin and S.P. Novikov, Sov. Math. Dokl. 27 (1983) 665 .

[14] J. Weiss, J. Math. Phys. 25 (1984) 2226.

[15] Ö. Sarıoglu, thesis (unpublished).

[16] I.Ya. Dorfman and O.I. Mokhov, J. Math. Phys. 32 (1991) 3288.

[17] B.A. Kupershmidt, Phys. Lett. A 123 (1987) 55.

[18] P.J. Olver, J. Math. Phys. 18 (1977) 1212. 